Bator Damian, Jańczyk Magdalena, Samek Ilona, Wójcik Magdalena, Dąbrowska Justyna, Lopuszańska Urszula. Effectiveness of Equine Assisted Activities or Therapies in relation to mental illness and mental disorders - a review. Journal of Education, Health and Sport. 2020;10(6):309-320. eISSN 2391-8306. DOI http://dx.doi.org/10.12775/JEHS.2020.10.06.033

https://apcz.umk.pl/czasopisma/index.php/JEHS/article/view/JEHS.2020.10.06.033

https://zenodo.org/record/3923186

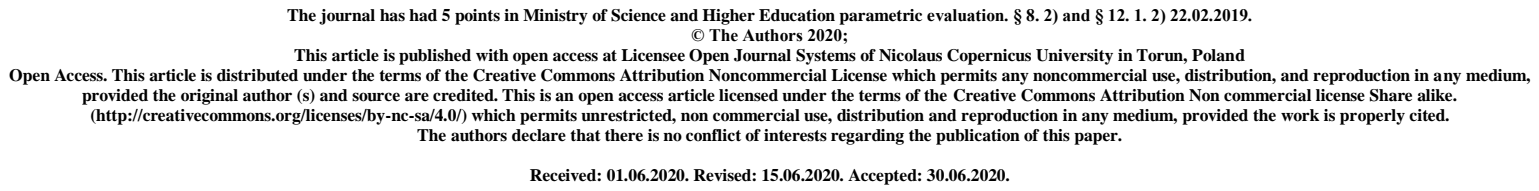

\title{
Effectiveness of Equine Assisted Activities or Therapies in relation to mental illness and mental disorders - a review
}

\author{
Damian Bator, Magdalena Jańczyk, Ilona Samek, Magdalena Wójcik, \\ Justyna Dąbrowska, Urszula Lopuszańska
}

Damian Bator $^{1}$, 0000-0002-8464-932X, bat199979@gmail.com

Magdalena Jańczyk ${ }^{1}$, 0000-0002-4948-9463, magdajanczyk7@ gmail.com

Ilona Samek ${ }^{1}$, 0000-0002-5538-5396, samekilona@gmail.com

Magdalena Wójcik ${ }^{1}$, 0000-0002-0999-6284, magdalena.wojcik967@gmail.com

Justyna Dąbrowska ${ }^{1}$, 0000-0002-1356-6965, jdabrowska00@gmail.com

dr n. med. Urszula Łopuszańska ${ }^{2}$, 0000-0001-6517-7577, urszula.lopuszanska@umlub.pl

1) Student Scientific Circle at the Department of Applied Psychology of the Medical University

in

Lublin

2) Department of Applied Psychology, Medical University of Lublin

\section{Summary}

Introduction and purpose: the Terminology Guidelines of the American Hippotherapy Association defines Equine Assisted Activities or Therapies (EAA/T) as a collection of different forms of contact between man and horse. EAA/T have become increasingly common interventions in the last decade, demonstrating therapeutic benefits in various areas of mental health. The aim of this report is to identify the forms and programmes of EAA/T that are used and to summarise and assess the latest available evidences of their clinical effectiveness in the treatment of symptoms of mental illness and disorders such as autism spectrum disorders, 
post-traumatic stress syndrome or schizophrenia. In order to achieve the above mentioned objectives, a literature review has been conducted to summarise existing evidence relating to the subject matter of the study in a descriptive manner. Description of the state of knowledge: 13 findings were found in autism spectrum disorders (1 review paper and 5 research papers), post-traumatic stress syndrome (1 review paper and 4 research papers), schizophrenia (1 review paper), and children whose parents were addicted (1 research paper). Summary: the results of the research indicate that Equine Assisted Activities and Therapies (EAA/T), including horse riding, may be beneficial for people with mental illnesses and disorders, such as autism spectrum disorders, post-traumatic stress syndrome, schizophrenia, and for people whose parents were addicted. The promising results so far support further research projects.

Key words: hippotherapy; equine assisted; mental illness

\section{Introduction and purpose}

In the Polish literature, equine assisted therapies and activities are described by the term hippotherapy, which is defined as therapeutic activities aimed at improving human functioning in physical, emotional, cognitive and/or social spheres, during which a specially prepared horse is an integral part of the therapeutic process (Gasińska Monika, Krupiński Jan and Paszkiewicz Alicja, Smolak Wincenty, Solecka Irena, Strumińska Anna, Ustjan Dominika, 2002). Among the indications there are mental illnesses both among adult and paediatric patients (Gasińska Monika, Krupiński Jan and Paszkiewicz Alicja, Smolak Wincenty, Solecka Irena, Strumińska Anna, Ustjan Dominika, 2002). The Terminology Guidelines of the American Hippotherapy Association defines Equine Assisted Activities or Therapies (EAA/T) as a collection of different forms of contact between man and horse and recommends distinguishing Equine Assisted Activities (EAA) and Equine Assisted Therapies (EAT) (Terminology and Horsemanship, 2018). An EAA is an activity accompanied by a horse, whether or not involving horseback riding, where the emphasis is on the participant's equestrian skills. In addition, EAAs support the education and recreation process and develop physical activity and recreation skills. Examples of activities may include equine assisted learning (EAL), adaptive riding (AR), horseman's skills, stable management, etc. Equine Assisted Therapies (EAT) is a term used to describe a group of therapies provided by licensed health care professionals that include horses and/or interactions with horses as a treatment tool (Terminology and Horsemanship, 2018). EAA/T have become in the last decade increasingly widespread interventions that have shown bio-psychosocial benefits and therapeutic outcomes in different areas of mental health including depression, anxiety, self-efficacy and self-esteem (Jormfeldt and Carlsson, 2018). 
The aim of this report is to identify the forms and programmes of EAA/T used, and to summarise and evaluate the latest available evidence on their clinical effectiveness in treating the symptoms of mental illness and disorders such as: autism spectrum disorders (ASD), posttraumatic stress syndrome (PTSD), or schizophrenia.

A review of publications on the effectiveness of EAA/T programmes in the treatment of mental illness and disorders has been carried out, according to the scheme described below.

Stage one: Search procedure

In spring 2020, we performed a search in the PubMed database (NCBI). The strategy was to search for articles in English published in the years 2015-2019 using the descriptors "animal asistedassisted therapy equine". The search resulted in 109 results.

Stage 2: Application of inclusion and exclusion criteria

First, we applied the following inclusion criteria:

1) the work only concerned EAA/T

2) the works were of an review or original character

3) the work focused on the clinical effects of EAA/T on patients with a specific psychiatric disease or disorder or on the mental sphere of the patients mentioned.

In the second stage, we applied an additional exclusion criterion. Documents were excluded if they presented case studies or series of cases below 10 . The 13 results remaining after selection were integrated into one Mendeley library, which facilitates efficient organization and management of references.

Stage 3: Extracting data

All selected articles have been read in full in order to extract data related to patient diagnosis, study chatter, sample size, form and EAA/T results.

\section{Autism spectrum disorder}

B. McDaniel Peters, and Wood have reviewed a thirty-three studies of children and adolescents with ASD that were published in English between 1980 and 2015. 25 out of 33 studies described research programmes classified as EAA (therapeutic riding TR, psycho-educational horseback riding (PER), community-based therapeutic recreation (CTR), equine-facilitated learning programme (EFL) riding for the disabled programme (RDA), grooming the horse (GRM), while the remaining 8 studies were conducted as EATs (hippotherapy (HPOT), short-term equine-assisted therapy (ST-EAT), simulated developmental horse-riding (SDHR) and unspecified EAT). There was significant variation between five different horse-assisted activities and four different horse-assisted therapies. Thirty-two out of thirty-three studies (97\%) were quantitative. Only one study (3\%) of the EAA used mixed method design with qualitative methods (Erdman et al. 2015).

In 25 EAA studies the subject of the measurements were:

- behaviour - 11 studies (44\%), of which nine (82\%) showed an improvement in at least one aspect, namely the child's riding behaviour and negative behaviour after the intervention. Behaviour was most often measured using structured observations or the Aberrant Behavior Checklist Community (ABC-C) scale.

-Interpersonal interactions - 10 studies (40\%), of which nine (90\%) showed improvement in one or more areas of this aspect. The most common evaluation of results used to measure interpersonal interactions was the Social Responsiveness Scale (SRS). 
-Communications - 9 studies (36\%), of which eight (89\%) reported improvement in one or more areas of communication. Communication was most often measured through behavioural observations and the Vineland Adaptive Behavioural Scale, second edition (VABS-II).

-Sensory integration - 7 studies (28\%), of which five (71\%) reported improvement. Sensory integration was most often measured with a sensory profile.

-Voluntary movement control - 6 surveys (24\%), of which four (67\%) reported improvement. Voluntary movement control was most often measured with the Bruinks-Osteretsky test (BOT-2).

-Indicators of the severity of autism syndrome - 4 studies (16\%) in which there was an improvement measured mainly with the Gilliam Autism Rating 2 scale

-quality of life - 4 studies $(16 \%)$ in which there was an improvement.

In 8 studies concerning EAT, the subject of measurements were:

- voluntary traffic control - 5 studies (63\%), which showed improvement.

-Self-care - 2 studies (25\%), which showed a statistically significant improvement.

Additionally, among the EAT studies, single studies measured: indices of severity of autism symptoms, involvement during play, cortisol in saliva, progesterone. In all these studies an improvement in results was noted.

In total, 114 different measurements were identified and organized in 33 studies.

The most common measurements concerned part of the first International Classification of Functioning, Disability and Health (ICF), i.e. functioning and disability, and more specifically its two components

-Activity and participation (ICF-AP) - 37\%

-Body functions and structures (ICF-BF) - 30\%

$7 \%$ of all results were classified as indicators of severity ASD. The remaining $26 \%$ of the measurements were classified as different.

Altogether across the 33 studies authors tended to report positive results, and $45 \%$ of all results were reported as statistically significant (McDaniel Peters and Wood, 2017).

Sudha et al. presented an overview that largely coincides with the above mentioned work. (Srinivasan et al., 2019).

Research work found

A, Harris and J. Williams presented a study on the intervention of horseback riding for children with ASD. A sample of 26 patients with autism spectrum disorders aged 6 to 9 years was assigned to the intervention, where the study group was 12 and the control group was 14 . Two-thirds of the participants communicated non-verbally and about one-third of them showed difficulties. Children were assigned to an intervention or control group according to their class membership: two classes were directed to equestrian activities and two remained in the control group.

Riding interventions consisted of 45-minute lessons conducted once a week for 7 or 5 weeks. The classes were composed of a 15-minute preparatory part and 30 minutes of different equestrian activities. During the EAA/T most of the children were supervised by a riding instructor who conducted the sessions. At the same time the control group had no occupational therapy. All participants received logopedic therapy for at least half a day a week, during the whole school stay. 
The pre- and post-treatment measures were carried out using the Children's Autism Evaluation Scale (CARS2) and the Aberrant Behaviour Checklist ABC-C.

After the study, a significant reduction in the severity of ASD symptoms, measured on both CARS2 and ABC-C scales, was found only for the intervention group. There were no significant changes in the level of sleepiness, irritability, stereotype and inappropriate speech of participants after the intervention period. (Harris and Williams, 2017).

Researchers at the School of Psychology at Lincoln University have assessed the impact of a 5-week therapeutic driving program consisting of 3-hour sessions on the social functioning of patients with autism spectrum disorders.

Stud staff were specially trained to work with children with disabilities and special needs. Participants and guardians were obliged to fill in forms concerning responsibility and health and safety at work. The research group consisted of 15 patients aged between 5-16 years with diagnosed ASD. The selection of participants was based on attending a school adapted to the requirements of children and young people with ASD. The study did not include a control group. The study pointed to the co-occurrence of double diagnosis (ADHD, sensory integration disorders) among some patients.

The effectiveness of the programme was assessed using the following tools: the autism spectrum quotient, the Vineland Adaptive Behaviour Scale VABS and the empathising and systematising quotient, which were filled in by caregivers at the beginning and end of the therapeutic programme. The results of the study showed that the TR intervention increased empathy and reduced adverse behaviour. The results also showed that the intervention did not affect specific adaptive behaviours such as socialization and communication (Anderson and Meints, 2016).

Multicentre Italian study under the leadership of Borgia and Loliva studied the effectiveness of EAT in improving functioning in children with ASD. Children were recruited for the study from the paediatric neuropsychiatric wards in Rome, and the following recruitment criteria were met: diagnosis of autism spectrum disorders, age 6-12 years, intelligence quotient $>70$ (according to WISC-III), lack of experience with hippotherapy, written consent of caregivers. A group of 28 recruited patients was randomly assigned to the study group (15 persons) and control group (13 persons). The therapy programme consisted of 25 weekly sessions, lasting 60-70 minutes, conducted in groups of 3-4 people. The therapy sessions consisted of organized activities with the participation of horses and included both grooming, walking with horses and horseback riding. Horse riding was introduced during the fifth session. The therapeutic team consisted of a FISE-certified riding instructor and a doctor (present at least once during the 8th session). Visual aids (pictures/colour drawings and posters) depicting the horses, and tools for horse care were kept in the stable during the whole session. Similar drawings were given to families of children so that they could colour them at home. Each of the subjects was evaluated before the start of the study and after 6 months (within 30 days after the end of therapy sessions), using two scales: The Vineland Adaptive Behavior Scale VABS and the Tower of London (TOL) scale. The study noted a time-dependent improvement in the performance of children with ASD attending EAT sessions (compared to the control group), namely an increase in improvement rates) in the field of socialisation on 
the VABS scale, and improvements in planning and problem-solving aspects on the TOL scale (Borgi et al., 2016).

Researchers from the University of Anschutz Medical Campus of Colorado and the Children's Hospital of Colorado have carried out an interventional study with horses to assess the effectiveness of therapeutic horse riding in terms of socialisation, communication, adaptive and motor behaviour in children with ASD. The participants were recruited using leaflets distributed to hospitals associated with universities, schools and organizations supporting parents of children with ASD. 127 ASD patients aged 6-16 years were recruited and stratified for non-verbal IQ tests $(\leq 85$ or $>85$ ) and randomly assigned to the study and control groups. The intervention group was included in a 10-week programme consisting of weekly 45 minute individual riding lessons, which also used behavioural teaching methods adapted to patients with ASD (e.g. using visual aids, praising appropriate behaviour and using participant interest to support engagement). Participants assigned to the control group were directed to work in a stable with a prior proposal of free riding lessons after the study was completed. The members of the control group had no contact with horses, but were also involved in activities conducted by a riding instructor and therapist with experience of working with children with ASD. The classes consisted of learning how to take care of the coma, in which a stuffed horse of natural size was an integral part of the teaching.

During the month before the start of the study, and after its completion, a number of tests were conducted to assess the level of participants' functioning. The following tools were used:

1. Peabody Picture Vocabulary Test (Peabody Picture Vocabulary Test PPVT-4)

2. Systematic Analysis of Language Transcripts (SALT)40)

3. Bruinks-Oseretsky Test of Motor Proficiency-2nd Ed (BOT-2)41)

4. Sensor Integration and Praxis Test (SIPT)42)

5. Vineland Adaptive Behavior Scale VABS

6. Aberrant Behaviour Checklist ABC-C36

7. The Social Responsiveness Scale (SRS)

The participants of the large sample achieved much greater improvement in the areas measured with the given tools: self-control (ABC-C)36, socialisation (SRS)47, communicativeness (SALT)40. The high degree of improvement was also observed in the areas of irritability, hyperactivity, social communication, number of spoken words and number of new words. However, there were no statistically significant differences between the groups in relation to the other measures, including adaptive (VABS-II)43 and motor behaviour (BOT-2 and SIPT),41.42 and PPVT-4.39 (Gabriels et al., 2015).

It is also worth quoting a study derived from the above, which refers to the same therapeutic programme in the same group of patients. This study presents additional results on the interaction of study subjects with their pet(s). The nature of the diagnostic features of ASD may jeopardise the interaction between children with ASD and their pets, which is reported as a problem by caregivers. It was assumed that participants in horse-riding therapeutic interventions will show an increase in positive interactions with their pets compared to control subjects. In order to determine the truthfulness of this hypothesis, out of the 127 individuals enrolled in the first study, 67 were selected and included in the additional study if the caregivers found that at least one pet lived at home. 31 patients were included in this study 
and 36 patients in the control group. The method of group randomization and therapeutic program was the same as in Gabreils et al. (2015). The caregivers were asked to fill in the modified for the study questionnaire "Child's attitude and behaviour towards animals". ( Child's Attitude and Behaviour toward Animals CABTA ), on the basis of which two scales were determined: Animal attachment score AATS and animal abuse score AABS. The questionnaires were completed as part of a larger study with a set of other tests within one month before and after the study. The AATS score of participants in the Therapeutic Horse Riding (THR) group improved significantly after the intervention, while the BA score did not show a trend towards improvement. No statistically significant differences were observed between the groups in the baseline change and after the intervention in the AABS (Petty et al., 2017).

\section{Posttraumatic stress disorder (PTSD)}

Review work found

$\mathrm{EAA} / \mathrm{T}$ for the civilian population with diseases and disorders that often also affect war veterans have shown promising results suggesting that they can bring similar benefits also among war veterans. The aim of this review was to describe the literature published so far on EAA/T in relation to war veterans (Kinney et al., 2019).

Searched works published between 1980 and 2017 with 3336 unique articles. These were subsequently reselected to capture peer-reviewed original research papers published in English that focused primarily on EAA/T for a population of which at least $80 \%$ were war veterans. The application of these criteria further narrowed the database to 6 research papers. Four of the six studies included interventions classified as EAA (THR, equine-assisted learning (EAL) and EAA unspecified) and two EAT programmes in the form of hippotherapy (HPOT) and Equine facilitated mental health (EFMH).

Although these studies had a quasi-experimental designs, two of them had relatively large sample sizes (58 and 51 participants), while the others had relatively small sample sizes (from one to 13 participants). While all six studies analysed the results quantitatively, two studies used a mixed approach, in these six studies the measurements were classified as follows:

-3 results were classified as ICF-AP

-4 results which are classified as ICF-BF

All six studies included at least one result classified as different, and the results varied greatly. Results classified as "other" included quality of life (three surveys), disability (two surveys), social support (one survey), resilience, self-learning and feelings related to the intervention itself.

Thirteen results classified as "other" were also generally positive, with two results reflecting statistically significant findings, seven results considered promising and the other four negative. Five out of six studies showed results that were classified as relevant for the DSM-5 classification. Of these five studies, PTSD symptoms and depressive symptoms were the most commonly addressed (three studies each). Studies that evaluated DSM-5-compliant parameters showed positive results, with one of nine results reflecting a statistically significant reduction in PTSD symptoms. The remaining eight DSM-5-compliant results were considered promising (Kinney et al., 2019). 
Research work found

A multi-centre Australian study assessed the results of the ST-EAT for veterans of the armed forces and their partners in terms of depression, anxiety, stress, post-traumatic stress, happiness and quality of life, and compared the results of the individual and couple programmes. The participants were members of the Veterans Service, Mates4Mates, who identified themselves as injured or ill, and a total of 47 veterans and partners from both the individual programme $(n=25$; veterans only) and the couple programme $(n=22)$. As the study is an evaluation of the existing programme offered by the Veterans' Service Organisation, it was not possible to randomly assign to the state of a control group or a naturally occurring control group that could be compared. The ST-EAT programme was a five-day inpatient therapy course. Based on the EAL/EAP Model of the Institute of Horse Psychotherapy in Australia, the programme included "Relational Gestalt Therapy", meditation sessions (mindfulness) to learn riding skills (which were not horse riding). All activities during the sessions were based on the learning of new skills in order to trigger social involvement of participants. Additionally, group discussions were arranged to give participants an opportunity to reflect on their experiences from the classes. The programs are conducted in individual formulas and couples (military veterans with their partners). The couples' program included the same therapeutic activities as the individual program, but was enriched with dating and counselling for couples to improve communication skills and build trust and respect. Measuring tools used included the Depression and Anxiety Stress Scale, the Posttraumatic Stress Disorder Control List for DSM-5, the Oxford Happiness Questionnaire, and the Quality of Life and Satisfaction Questionnaire. A psychometric evaluation was conducted before the start of the horse-assisted therapy program, immediately after the program ends, and three months after the program ends. Statistical analysis showed that both individual and pair programmes had significantly fewer symptoms and significantly higher levels of happiness and quality of life after the intervention compared to the previous state. The reduction of symptoms persisted during the three months of observation only for participants of the pair programme. Participants in the couple program reported significantly fewer symptoms of depression, PTSD at the time of observation compared to participants in the individual program (Romaniuk, Evans and Kidd, 2018).

A US multicentered study to test the effectiveness of a 6-week THR program in reducing PTSD symptoms and increasing self-sufficiency, emotional control, social and emotional loneliness used a standardised study with a control group. Recruitment took place by sending invitations to veterans, advertising in a spike, and referral by clinicians. The veterans who were contacted by the research office to report their participation in the project. Other inclusion criteria were: age at least 18 years, no longer in active military service, body mass up to $200 \mathrm{~kg}$, ability to walk at least 7.6 meters independently and willingness to interact and ride. 57 participants were recruited and 29 enrolled in the study in a randomized manner. They were randomly assigned either to an equestrian group $(n=15)$ or to a waiting list control group $(n=14)$. The waiting list control group survived a 6 week waiting period while the equestrian group started THR. Participants participated in THR classes once a week for 6 weeks according to a structured THR program developed by the research team, in which 2 occupational therapists and 2 certified riding instructors participated. During the THR session, 
war veterans learned basic riding skills as well as riding for 55-70 minutes. PCL-M scales and other tools were used to measure wellbeing and symptoms of PTSD, including Coping Self Efficacy Scale (CSES), Difficulties in Emotion Regulation Scale (DERS) and Social and Emotional Solitude Scale for the short version for adults (SELSA). In the subjects there was a statistically significant decrease in PTSD scores after 3 weeks of THR $(\mathrm{P} \leq 0.01)$ and a statistically and clinically significant decrease after 6 weeks of THR $(\mathrm{P} \leq 0.01)$. There was $66.7 \%$ probability of obtaining lower PTSD scores after 3 weeks and $87.5 \%$ after 6 weeks. The results concerning self-sufficiency, emotional control and social and emotional solitude did not achieve statistical significance. The results concerning self-sufficiency and emotional control showed a tendency in the predicted direction, in comparison to the results of emotional solitude (Johnson et al., 2018).

2.2.2.3. The aim of the study carried out by researchers from the University of New Mexico is to carry out a twin project for the study mentioned above, i.e. to investigate the impact of the EAP on the symptoms of PTSD. It was assumed that veterans with PTSD who participate in the standardized EAP for $1 \mathrm{~h}$ per week for 6 weeks will experience reduced symptoms of PTSD and show increased immunity compared to people who do not receive EAP interventions. A sequential, two-armed, parallel group study was conducted comparing 6 weeks of EAP with standardized, predetermined, ongoing PTSD therapy. The therapy was carried out in a social EAP centre in cooperation with an academic university hospital. Twenty adult veterans with symptomatic PTSD completed the study. The inclusion criteria for participants included veterans with existing PTSD diagnosis, who scored at least 29 points on the PCL-M scale. Exclusion criteria included adults who were unable to give informed consent, people under 18 years of age, pregnant women, prisoners, those receiving antipsychotics and those receiving glucocorticoid therapy. Ten adult veterans with prediagnosed PTSD were assigned to the EAP and received targeted interactions with trained horses for one hour per week in groups of 3 or 4, while continuing with pre-determined therapy. The EAP did not cover horseback riding. The results were compared with the results obtained from 10 adult veterans, who received only standard, predetermined care of horses with PTSD. Measurements included: changes in salivary cortisol levels, results for the PCL$\mathrm{M}$ scale, and the Connor-Davidson Resistance Scale (CD-RISC) before and after the completion of programmes.

PCL-M scores decreased significantly in both groups, but there was no difference between the groups in terms of score reduction during the study. CD-RISC scores increased significantly in EAP group. There were no differences between the groups in terms of morning cortisol concentrations in saliva (Burton, Qeadan and Burge, 2019).

Researchers from the Department of Psychology, Florida Atlantic University have tested the effectiveness of the Equine Partnering Naturally approach to equine assisted treatment in treating post-traumatic anxiety and stress (PTSD). The participants were 16 volunteers who experienced a traumatic event such as rape or serious accident and had present symptoms of PTSD above the recommended limit of 31 in the PCL-S. The elapsed time from the injury was 1 to 39 years $(\mathrm{M}=19.36, \mathrm{SD}=13.35)$.

Participants were involved in horseback tasks in 2-hour sessions in three groups of five to six people. The participants came to the centre once a week for 6 weeks. 
Each two-hour session was led by a specialist in psychiatry and psychotherapy. In session 1, participants met with horses and worked with them to develop self-awareness, improve concentration and improve listening skills. In session 2, participants learned how to conduct non-verbal interactions with horses, examined the effects and body language. In session 3, participants learned how to hold back horses and worked on coping with challenges and stressful situations. In session 4 participants learned to guide and support the horse. They were involved in creating safe spaces and marking out boundaries in relations. In session 5, participants learned how to stay focused in the face of distraction or temptation. During 6 sessions, participants tested their previously acquired skills and worked on inner peace and stability. This program was considered a full course of treatment.

The measurement tools used were: PCL-S scale, LEC scale for injury history, Trauma Emotional Trauma Inventory, Generalised Anxiety Disorder Scale (GAD-7), Patient Health Questionnaire (PHQ-9) measuring depression, Alcohol Use Disorder Identification Test (AUDIT), Patient Health Questionnaire (PHQ-15) measuring severity scales of somatic symptoms, Five-Dimensional Validity Questionnaire (FFMQ). The Inventory for Measuring Self-Consultation, Generalised Self-Efficacy Scale (GSE), Social Support Scale (SSS), Life Saving Disability Scale (SWLS). The questionnaires were provided within a few weeks before the first session, and immediately after the final session.

Immediately after the final session, participants reported significantly reduced symptoms of post-traumatic stress, less serious emotional reactions to injury, less generalised anxiety and fewer symptoms of depression. In addition, participants improved self-awareness strategies, and reduced alcohol consumption. There was no significant impact of treatment on physical health, proactive coping, self-sufficiency, social support or life satisfaction (Earles, Vernon and Yetz, 2015).

\section{Schizophrenia}

The aim of the work of Jormfeldt and Carlsson was a systematic review of EAA/T studies among adults with diagnosed schizophrenia. The qualitative and quantitative empirical reviewed papers published in English in the period from 2000 to 2016, which concerned people aged 18-65 years with diagnosed schizophrenia who participated in EAA/T, were identified as important criteria for publication in accordance with the research objective.

The presented results consist of six articles, four qualitative and two quantitative, published in international scientific journals. In total, the articles cover 137 individuals (between 5 and 90 patients in each study) diagnosed with schizophrenia or related disorders, who participated in $\mathrm{EAA} / \mathrm{T}$.

Among the six reviewed articles, participants received various forms of EAA/T focusing on horse riding and activities forming a bond between the horse and the patient. In individual studies there were also such activities as horse care or work in a stable. Apart from therapeutic activities related to horses, the research could include additional therapeutic content. They were also varied in terms of the duration of the sessions and the people who conducted them. 4 studies included EAA, where sessions were held once a week between 10 weeks and 6 months, and 2 EAT studies, where sessions were also held once a week (for 24 months or 10 weeks). 
None of the studies on therapy showed any negative results associated with interventions. These studies showed different types of positive results depending on the purpose of the study and the study design. Only two of the six studies included were assessed as having high quality evidence.

The results of this review indicate that horse-assisted interventions can facilitate the recovery of people suffering from schizophrenia by stimulating them to take an active role in their own rehabilitation (Jormfeldt and Carlsson, 2018).

Children Exposed to Problematic Parental Substance Use

Evidence suggests that problematic alcohol consumption and drug use can reduce parental skills. Children at risk of problematic parental use (PPSU) often face many harmful effects on development. Researchers at the University of Griffith School of Services and Social Work implemented a 12-week EAT program consisting of 2-hour weekly sessions attended by 41 children (average age 10.26). A total of five therapeutic groups were included in the study, with participants ranging from 5 to 10 children. Sessions were divided into five themes: care, connection with readiness, cooperation and celebration. Each therapeutic session was conducted by a qualified horse trainer. A specialist trained by EAT in psychology, social work and treatment of alcohol and other drugs addiction worked in the EAT programme. Parents of children $(n=41)$ and school teachers $(n=31)$ completed the Force and Difficulty Questionnaire (SDQ) before and after the intervention. The parents reported that after the 12week EAT programme the problems with children's behaviour, emotional problems, hyperactivity and peer problems decreased, while the pro-social behaviour of the children improved (Tsantefski et al., 2017).

\section{Conclusions}

The results of the research indicate that Equine Assisted Activities and Therapies (EAA/T), including horseback riding, can be beneficial for people with mental illnesses and disorders, such as autism spectrum disorders, post-traumatic stress syndrome, schizophrenia, and for people whose parents were addicted. The review suggests that further research is needed to establish a solid evidence base for EAA/T among people with these problems. The promising results so far support further research projects. There is also a need for more systematic, staged research that will develop and evaluate the individual components of the intervention, closing gaps in knowledge or research approaches. Increased knowledge of these phenomena will facilitate the identification of those interventions which are most effective.

\section{List of references}

Anderson, S. and Meints, K. (2016) 'Brief Report: The Effects of Equine-Assisted Activities on the Social Functioning in Children and Adolescents with Autism Spectrum Disorder', Journal of Autism and Developmental Disorders. Springer US, 46(10), pp. 3344-3352. doi: 10.1007/s10803-016-2869-3.

Borgi, M. et al. (2016) 'Effectiveness of a Standardized Equine-Assisted Therapy Program for Children with Autism Spectrum Disorder', Journal of Autism and Developmental Disorders. Springer US, 46(1), pp. 1-9. doi: 10.1007/s10803-015-2530-6.

Burton, L. E., Qeadan, F. and Burge, M. R. (2019) 'Efficacy of equine-assisted psychotherapy 
in veterans with posttraumatic stress disorder', Journal of Integrative Medicine. Shanghai Changhai Hospital, 17(1), pp. 14-19. doi: 10.1016/j.joim.2018.11.001.

Earles, J. L., Vernon, L. L. and Yetz, J. P. (2015) 'Equine-Assisted Therapy for Anxiety and Posttraumatic Stress Symptoms’, (April), pp. 149-152. doi: 10.1002/jts.

Gabriels, R. L. et al. (2015) 'Randomized Controlled Trial of Therapeutic Horseback Riding in Children and Adolescents With Autism Spectrum Disorder', Journal of the American Academy of Child and Adolescent Psychiatry. Elsevier Inc, 54(7), pp. 541-549. doi: 10.1016/j.jaac.2015.04.007.

Gasińska Monika, Krupiński Jan, N. M. and Paszkiewicz Alicja, Smolak Wincenty, Solecka Irena, Strumińska Anna, Ustjan Dominika, W. M. (2002) 'Kanony polskiej hipoterpii', pp. 18.

Harris, A. and Williams, J. M. (2017) 'The impact of a horse riding intervention on the social functioning of children with autism spectrum disorder', International Journal of Environmental Research and Public Health, 14(7). doi: 10.3390/ijerph14070776.

Johnson, R. A. et al. (2018) 'Effects of therapeutic horseback riding on post-traumatic stress disorder in military veterans', Military Medical Research. Military Medical Research, 5(1), pp. 1-13. doi: 10.1186/s40779-018-0149-6.

Jormfeldt, H. and Carlsson, I. M. (2018) 'Equine-Assisted Therapeutic Interventions Among Individuals Diagnosed With Schizophrenia. A Systematic Review', Issues in Mental Health Nursing. Taylor \& Francis, 39(8), pp. 647-656. doi: 10.1080/01612840.2018.1440450.

Kinney, A. R. et al. (2019) 'Equine-assisted interventions for veterans with service-related health conditions: A systematic mapping review', Military Medical Research. Military Medical Research, 6(1), pp. 1-15. doi: 10.1186/s40779-019-0217-6.

McDaniel Peters, B. C. and Wood, W. (2017) 'Autism and Equine-Assisted Interventions: A Systematic Mapping Review', Journal of Autism and Developmental Disorders. Springer US, 47(10), pp. 3220-3242. doi: 10.1007/s10803-017-3219-9.

Petty, J. D. et al. (2017) 'Therapeutic horseback riding crossover effects of attachment behaviors with family pets in a sample of children with autism spectrum disorder', International Journal of Environmental Research and Public Health, 14(3). doi: 10.3390/ijerph14030256.

Romaniuk, M., Evans, J. and Kidd, C. (2018) 'Evaluation of an equine-assisted therapy program for veterans who identify as "wounded, injured or ill" and their partners', PLoS ONE, 13(9), pp. 1-15. doi: 10.1371/journal.pone.0203943.

Srinivasan, S. M. et al. (2019) 'HHS Public Access', 5(2), pp. 156-175. doi: 10.1007/s40489018-0130-z.Effects.

Terminology, C. and Horsemanship, C. (2018) 'AHA, Inc Terminology Guidelines Recommended Terminology’, (December), pp. 1-12.

Tsantefski, M. et al. (2017) 'An open trial of equine-assisted therapy for children exposed to problematic parental substance use', Health and Social Care in the Community, 25(3), pp. 1247-1256. doi: 10.1111/hsc.12427. 